THEOREM V. (a) If $\left\{a_{n}\right\},\left\{\varepsilon_{n}\right\}$ are positive sequences such that $\varepsilon_{n} \searrow 0$, POLONICI MATHEMATICI XI (1961)

$\sum_{n=1}^{\infty} a_{n} \varepsilon_{n}$ converges, then $\sum_{n=1}^{\infty}(-1)^{n-1}\left(a_{1}+\ldots+a_{n}\right) \varepsilon_{n}$ converges.

(b) If $\left\{b_{n}\right\}$ is another positive sequence such that

$$
\varlimsup_{n \rightarrow \infty} \frac{b_{1}+\ldots+b_{n}}{a_{1}+\ldots+a_{n}}<\infty
$$

then $\sum_{n=1}^{\infty}(-1)^{n-1}\left(b_{1}+\ldots+b_{n}\right) \varepsilon_{n}$ also converges.

Proof. (a) follows from Lemma 3 with $u_{n}=(-1)^{n-1}\left(a_{1}+\ldots+a_{1 b}\right)$

(b) follows from (a) since $\sum_{n-1}^{\infty} b_{n} \varepsilon_{n}$ is convergent by Lemma 3 again.

Theorem $\mathrm{V}$ gives rise to the following result, proved by Th. Kaluza ([2], Satz 23) by a different method in the particular case $\lambda_{n}=n$.

CoRoLlary. If $\beta \geqslant 0$ and $\left\{f(n) / n^{\beta}\right\}$ is ultimately positive monotonic decreasing, or equivalently $\{f(n)\}$ is positive quasi-monotonic decreasing, and if $\sum_{n=1}^{\infty} f(n)$ is convergent, $\left\{\lambda_{n}\right\}$ is a sequence of positive integer's monotonic increasing and unbounded, then $\sum_{n=1}^{\infty}(-1)^{n-1} \lambda_{n} f\left(\lambda_{n}\right)$ is convergent.

Pro of. Recalling Remark 1 at the end of $\$ 2$, we have only to choose, in Theorem $\nabla$ as modified by that remark,

$$
\varepsilon_{n}=\frac{f(n)}{n^{\beta}}, \quad a_{n}=n^{\beta}, \quad b_{n}=\left\{\begin{array}{ccc}
\lambda_{m}^{\beta+1}-\lambda_{m-1}^{\beta+1} & \text { if } & n=\lambda_{m}, \\
0 & \text { if } & n \neq \lambda_{m} .
\end{array}\right.
$$

This choice is justified since, arguing as we did with (11), we find that (13) holds for the above $\left\{b_{n}\right\}$ as well as for the $\left\{b_{n}\right\}$ in (11).

\title{
References
}

[1] A. Alexiewioz, On Canchy's condensation theorem, Studia Math. 16 (1957), p. $80-85$.

[2] Th. Kaluza, Struktur und Eigenschaften mehrfach monotoner Folgen, Math. Z. 26 (1927), p. $345-364$

[3] C. T. Rajagopal, On some limit theorems, Amer. J. Math. 70 (1948), p. 157-166 and p. 908

[4] O. Szász, Collected mathematioal papers, Cinoinnati 1955.

RAMANUJAN INSTITUTE OF MATHEMATICS, MADRAS, INDIA

Reģu par la Rédaction le 10.9. 1959

\section{Perturbations non linéaires qui n'augmentent pas la croissance maximale des intégrales}

\author{
par Z. SzMyDT (Kraków)
}

1. Envisageons le système d'équations différentielles linéaires, écrit sous la forme vectorielle

$$
\frac{d x}{d t}=A(t): c
$$

et le système perturbé

$$
\frac{d y}{d t}=A(t) y+f(t, y) .
$$

On suppose que $A(t)$ est une matrice carrée dont les éléments $a_{i j}(t)$ sont des fonctions de $t$, continues pour $t \geqslant 0$.

Soit $X(t)$ la matrice vérifiant les conditions

$$
\frac{d X}{d t}=A(t) X, \quad X(0)=I \quad(I \text { matrice unité }) .
$$

Nous l'appellerons matrice fondamentale du système (1).

Soient $\|x\|$ et $\|X\|$ les normes du vecteur $x=\left(x_{1}, \ldots, x_{n}\right)$ et de la matrice $X=\left(x_{i j}\right)$, données respectivement par les formules

$$
\|x\|=\sum_{i}\left|x_{i}\right|, \quad\|X\|=\sum_{i, j}\left|x_{i j}\right| .
$$

Dans cette note nous allons considérer le problème suivant.

PROBLìne Q. Correspond-il à chaque intégrale $y(t)$ du système (2) au moins une intégrale $x(t)$ du système (1) telle que

$$
y(t)=x(t)+o(\|X(t)\|) \text { ? }
$$

Dans le cas où le problème $\mathbf{Q}$ admet une réponse affirmative, chaque intégrale $y(t)$ du système (2) vérifie la relation $\left(^{1}\right)$

$$
y(t)=O(\|X(t)\|)
$$

(1) Cette relation a été étudiée dans [2]. Nous reviendrons sur ce sujet dans le emme 2 et dans la remarque 1 de cette note. 
c'est-à-dire la croissance d'aucune intégrale du système (2) ne dépasse la croissance maximale des intégrales du système (1), déterminée par celle de la fonction $\|X(t)\|$. En particulier, il résulte de (4) que toutes les intégrales du système (2) sont bornées lorsque le sont celles du système (1).

Dans les théorèmes 1 et 2 de cet article nous donnons des conditions suffisantes pour que le problème $Q$ admette une réponse affirmative. Le théorème 1 généralise un résultat de $R$. Bellman [1] se rapportant au cas linéaire

$$
f(t, y)=[B(t)-A(t)] y
$$

(cf. Corollaire 3 ) et le théorème 2 constitue une extension d'un de nos résultats antérieurs concernant la stabilité [3] (cf. Corollaire 1).

Dans les démonstrations des théorèmes de cet article nous appliquerons la même méthode que dans [3].

2. Voici d'abord un lemme qui coïncide, dans l'hypothèse $\gamma(t)=$ const, avec le théorème $(3.2 . i)$ de $[2]\left({ }^{2}\right)$ et dont la démonstration ne diffère pas de celle $\mathrm{du}$ théorème cité.

LEMME 1. Supposons que les fonctions $u(t), \gamma(t), h(t)$, non négatives lorsque $0 \leqslant t<\infty$ et intégrables dans chaque intervalle fini $\langle 0, t\rangle$, vérifient l'inégalité

$$
u(t) \leqslant \gamma(t)\left[e+\int_{0}^{t} u(\tau) h(\tau) d \tau\right] \text { lorsque } t \geqslant 0,
$$

dans laquelle c désigne une constante non négative.

Alors

$$
u(t) \leqslant c \gamma(t) \exp \left[\int_{0}^{t} \gamma(\tau) h(\tau) d \tau\right] \text {. lorsque } t \geqslant 0 .
$$

LEMME 2. Soient $X(t)$ la matrice fondamentale du système (1) et $f(t, y)$ une fonction continue $\left(^{3}\right)$ lorsque $t \geqslant 0$ (y arbitraire). Supposons qu'il existe pour $t \geqslant 0$ une fonction non négative et sommable $g(t)$ telle que

Soit

$$
\begin{gathered}
\|f(t, y)\| \leqslant g(t)\|y\| \quad \text { lorsque } \quad t \geqslant 0, \\
\int_{0}^{\infty}\|X(t)\|\left\|X^{-1}(t)\right\| g(t) d t<\infty .
\end{gathered}
$$

$$
K=\exp \left[\int_{0}^{\infty}\|X(t)\|\left\|X^{-1}(t)\right\| g(t) d t\right] .
$$

(2) Les informations bibliographiques concernant ce théorème sont données à la page 35 de [2].

$\left({ }^{s}\right)$ Cf. remarque 3
Cela admis, chaque intégrale $y(t)$ du système (2) vérifie l'inégalité

$$
\|y(t)\| \leqslant K\|X(t)\|\|y(0)\| \text { lorsque } \quad t \geqslant 0 .
$$

Remarque 1. Le lemme 2 constitue une légère généralisation $d u$ théorème (6.2.iv) de [2], l'hypothèse: $\|X(t)\| \leqslant M,\left\|X^{-1}(t)\right\| \leqslant M, \int_{0}^{\infty} g(t) d t<\infty$ y étant remplacée par l'inégalité (6).

Démonstration du lemme $2\left(^{4}\right)$. Soit $y(t)$ une intégrale quelconque du système (2). On a alors l'identité

(8) $\quad y(t)=X(t)\left\{y(0)+\int_{0}^{t} X^{-1}(\tau) f[\tau, y(\tau)] d \tau\right\} \quad$ lorsque $\quad t \geqslant 0$

d'où, en vertu de (5), on obtient l'inégalité

$$
\|y(t)\| \leqslant\|X(t)\|\left\{\|y(0)\|+\int_{0}^{t}\left\|X^{-1}(\tau)\right\|\|y(\tau)\| g(\tau) d \tau\right\} \quad \text { lorsque } \quad t \geqslant 0 .
$$

En appliquant le lemme $1(u(t)=\|y(t)\|, \quad \gamma(t)=\|X(t)\|, \quad c=\|y(0)\|$, $\left.h(\tau)=g(\tau)\left\|X^{-1}(\tau)\right\|\right)$ on déduit de la dernière inégalité la suivante:

$$
\|y(t)\| \leqslant\|y(0)\|\|X(t)\| \exp \left[\int_{0}^{t}\|X(\tau)\|\left\|X^{-1}(\tau)\right\| g(\tau) d \tau\right] \quad \text { lorsque } \quad t \geqslant 0 .
$$

L'intégrale $y(t)$ étant arbitraire, le lemme 2 en résulte (cf. (7)).

THÉORÈme 1. Dans les hypothèses au lemme 2, à chaque intégrale $y(t)$ du système (2) correspond au moins un vecteur b tel que

$$
y(t)=X(t) b+o(\|X(t)\|) \quad \text { lorsque } t \rightarrow \infty .
$$

Le problème $Q$ admet donc une réponse affirmative.

Démonstration. En vertu de (5) et du lemme 2 on obtient l'inégalité

$$
\begin{aligned}
\int_{0}^{t}\left\|X^{-1}(\tau)\right\|\|f[\tau, y(\tau)]\| d \tau & \leqslant \int_{0}^{t}\left\|X^{-1}(\tau)\right\|\|y(\tau)\| g(\tau) d \tau \\
& \leqslant K\|y(0)\| \int_{0}^{i}\left\|X^{-1}(\tau)\right\|\|X(\tau)\| g(\tau) d \tau
\end{aligned}
$$

qui moyennant l'hypothèse (6), assure la convergence de l'intégrale

$$
\int_{0}^{\infty} X^{-1}(\tau) f[\tau, y(\tau)] d \tau \text {. }
$$

(4) Cette démonstration est tout à fait analogue à celle du théorème (6.2. iv) de [2], anssi bien qu'à celle d'un théorème de [3] (cf. [3], p. 235, Démonstration I.) 
En introduisant les notations

$$
\begin{aligned}
b & =y(0)+\int_{0}^{\infty} X^{-1}(\tau) f[\tau, y(\tau)] d \tau, \\
v(t) & =-\int_{t}^{\infty} X^{-1}(\tau) f[\tau, y(\tau)] d \tau,
\end{aligned}
$$

on peut écrire l'identité (8) sous la forme

$$
y(t)=X(t) b+X(t) v(t) .
$$

Notre théorème se trouve ainsi démontré, car $b$ est un vectemr constant et $v(t)$ est une fonction vectorielle qui tend vers zéro lorsque $t$ tend vers l'infini.

THéronìme 2. Soit $W(t)$ la matrice fondamentale du système.

$$
\frac{d w}{d t}=B(t) w
$$

Soient $f(t, z)$ une fonction vectorielle continue lorsque $t \geqslant 0$ (z arbitraire) et $h(t)$ une fonction sommable et non négative lorsque $t \geqslant 0$. Supposons satisfaites les conditions suivantes:

$$
\|f(t, z)\| \leqslant h(t)\|z\| \text { lorsque } t \geqslant 0, \quad \int_{0}^{\infty} h(t) d t<\infty,
$$$$
\text { (11) } \quad f[t, W(t) y]=W(t) f(t, y) \text { lorsque } t \geqslant 0 \text {. }
$$

Dans ces hypothèses, à chaque intégrale z $(t)$ du système

$$
\frac{d z}{d t}=B(t) z+f(t, z)
$$

correspond au moins un vecteur constant $b$ tel que

$$
z(t)=W(t) b+o(\|W(t)\|) .
$$

Le problème $Q$ relatif aux systèmes (9) et (12) admet donc uno réponse affirmative.

Démonstration. Considérons la transformation.

$$
\approx=W(t) y \text {. }
$$

On vérifie facilement qu'en vertu de (11) la nouvelle variable $y$ satisfait à l'équation

$$
\frac{d y}{d t}=f(t, y)
$$

En appliquant le théorème 1 , dans lequel $A(t)$ est une matrice nulle et $X(t)=I$, on conclut qu'à chaque intégrale $y(t)$ du système (14) correspond un vecteur constant $b$ tel que

$$
y(t)=b+o(1) \text {. }
$$

En vertu de (13) notre théorème en résulte.

3. Voici maintenant le corollaire 1 qui résulte immédiatement du théorème 2 et qui coïncide avec un de nos résultats antérieurs concernant le problème de la stabilité [3].

CoRollatre 1. Toutes les solutions du système (12) sont bornées torsque le sont celles du système (9) et lorsque $f(t, y)$ est une fonction continue pour $t \geqslant 0$, vérifiant les conditions (10) et (11).

Le corollaire 2 que nous allons énoncer maintenant est une conséquence immédiate du lemme 2.

CoRollamRe 2. Toutes les solutions du système (2) sont bornées lorsque te sont celles du système (1) et lorsque $f(t, y)$ est une fonction continue pour $t \geqslant 0$, vérifiant les conditions (5) et (6).

Remarque 2. En remplaçant dans le corollaire 2 l'hypothèse (6) par les suivantes:

$$
\int_{0}^{\infty} g(t) d t<\infty, \quad\left\|X(t) X^{-1}(\tau)\right\| \leqslant N \quad \text { lorsque } \quad 0 \leqslant \tau \leqslant t<\infty,
$$

$N$ étant constante finie, on arrive formellement à un autre résultat que nous avons établi dans [3].

Remarque 3. L'hypothèse de la continuité de la fonction $f(t, y)$ pour les valeurs $t \geqslant 0$ ( $y$ arbitraire), introduite dans les théorèmes de cette note, peut être remplacée par une hypothèse plus faible, à savoir celle que toute solution $y(t)$ du système (2) vérifie l'équation (8).

Voici encore le corollaire 3 qui résulte immédiatement du théorème 1 et qui coincide avec le théorème de R. Bellman démontré dans [1].

CoRoLLAIRE 3. Soit $B(t)$ une matrice carrée dont les éléments sont des fonctions continues de $t$ pour $t \geqslant 0$. Supposons que

$$
\int_{0}^{\infty}\|B(t)-A(t)\|\|X(t)\|\left\|X^{-1}(t)\right\| d t<\infty,
$$

oì $X(t)$ désigne la matrice fondamentale du système (1).

Dans ces hypothèses, chaque solution $y(t)$ du système

$$
\text { peut être écrite sous la forme } \frac{d y}{d t}=B(t) y
$$

$$
y(t)=X(t) b+o(\|X(t)\|) \quad \text { lorsque } \quad t \rightarrow \infty .
$$




\section{Travaux cités}

POLONICI MATHEMATICI

XI (1961)

[1] R. Bellman, On a generalization of a resull of Wintner, Quarterly of Applied Mathematics 16 (1959), p. 431-432.

[2] L. Cesari, Asymptotic behavior and stability problems in ordinary differential equations, Springer-Verlag 1959

[3] Z. Szmydt, Sur les systèmes d'équations difjérentielles dont toutes les solutions sont bornées, Annales Polonici Mathematici 2 (1955), p. 234-236.

INSTYTUT MATEMATYCZNY POLSKIEJ AKADEMII NAUK

INSTITUT MATHEMATIQUE DE L'ACADEMIE POLONAISE DES SCIENCES

Regu par la Rédaction le 1. 10. 1959

\section{Sur l'existence des solutions périodiques de l'équation \\ différentielle $x^{\prime \prime}+f\left(x, x^{\prime}\right) x^{\prime}+g(x)=p(t)$}

\section{par Z. OpIaL (Kraków)}

1. Le but de la présente note est de montrer comment les théorèmes sur l'existence des solutions périodiques de l'équation différentielle $d u$ second ordre

$$
x^{\prime \prime}+g(x)=p(t),
$$

établis dans [1], s'étendent partiellement à l'équation différentielle plus générale

$$
x^{\prime \prime}+f\left(x, x^{\prime}\right) x^{\prime}+g(x)=p(t) .
$$

Supposons une fois pour toutes que les fonctions continues $f(x, y)$, $g(x)$ satisfassent aux conditions suivantes:

$$
\begin{gathered}
f(x, y) \geqslant 0, \quad \text { quels que soient } x \text { et } y, \\
x g(x)>0, \quad \text { quel que soit } x \neq 0, \\
\lim _{|x| \rightarrow \infty}|g(x)|=+\infty
\end{gathered}
$$

et admettons que la fonction $p(t)$ soit continue et périodique de période $\omega>0$ :

(6)

$$
p(t+\omega)=p(t), \quad \text { quel que soit } t .
$$

Posons de plus

$$
G(x)=\int_{0}^{x} g(u) d u \quad(-\infty<x<+\infty)
$$

et, pour tout $x \neq 0$, introduisons la fonction

$$
T(x)=\frac{1}{\sqrt{2}}\left|\int_{0}^{x} \frac{d u}{\sqrt{G(x)-G(u)}}\right| .
$$

En vertu de (4), pour tout voisinage fermé $V_{x}$ d'un point $x \neq 0$ qui ne contient pas l'origine, la fonction $g(u)$ est en valeur absolue supérieure à une constante, soit $m\left(V_{x}\right)$. On a donc

$$
|G(x)-G(u)| \geqslant m\left(V_{x}\right)|x-u| \quad u \in \nabla_{\boldsymbol{x}},
$$

